\title{
Higher Education "Quantity-Quality-Effect" and Fitness Landscape of Trusted Cooperation
}

\author{
Lin Jiang \\ Center for Higher Education and Management Research \\ Liaoning Police College, \\ Liaoning, Dalian, 116036, China
}

\author{
Ping $\mathrm{He}^{*}$ \\ Center for Higher Education and Management Research \\ Liaoning Police College \\ Liaoning, Dalian, 116036, China
}

\begin{abstract}
Based on the theory of synergetics and selforganization, a new research field of higher education system is proposed, which is the theory of higher education credible collaboration. Focusing on the effectiveness of two themes of "scale" (short for quantitative effect) and "quality effectiveness" (short for quality effect), the paper discussed the interdependence and mutual influence of higher education system, as well as " quantitative effect " and " quality effect ". The comprehensive criterion of " quantity-quality-effect " can fully reflect the operation state of higher education system. The research shows that the core issue of "quantity-quality-effect" of higher education is to seek a system mechanism of credible collaboration, whose operation state is studied by meso-scale method, and can be depicted by the adaptive landscape satisfying the collaborative development of society and education.
\end{abstract}

Keywords-higher education; quantity-quality-effect; trusted cooperation; fitness landscape

\section{INTRODUCTION}

The history of human development shows that education is the foundation of social development, and higher education is the catalyst of social development. At the first world conference on higher education held by UNESCO in 1998, it was stated that "we reaffirm that the basic mission and important role of higher education should be maintained, strengthened and further expanded, especially the mission of promoting sustainable development and progress of society as a whole". (1998, Paris: higher education in the 21st century: world declaration on vision and action) this declaration is also the common concept and goal pursuit of higher education in the 21 st century. It is also pointed out in the national medium and long-term education development plan that "higher education undertakes the major tasks of training senior specialized personnel, developing science, technology and culture, and promoting modernization. Improving quality is the core task of higher education development and the basic requirement of building a strong country in higher education. By 2020, the structure of higher education will be more rational, with more distinctive features, and the overall level of personnel training, scientific research and social services will be improved. ." (Outline of the national program for medium and long-term education reform and development, 2010).

It can be found that the reform and development of higher education has become an important historical mission of social and economic development. In recent years, a lot of research literature has been conducted on the concept, management mode and system, discipline and specialty setting, enrollment and employment of higher education, providing valuable research results for the reform and planning of higher education [1-6]. But, the traditional higher education research, in concept, management mode and system of higher education, disciplines and specialties, limited to the recruitment of students employment of Angle factors of education itself, without considering the differences of social fields on attribute education, lack of higher education science itself and human behavior characteristics and the relationship between social attribute analysis, at the same time, as a result of these studies is full of all kinds of uncertain factors in the elements, so the results can only be relative significance. Many research results show that the evaluation of higher education level is not only determined by the single concept of the quantity, quality and benefit of higher education, and how to evaluate the level of higher education scientifically, reasonably and effectively has not yet obtained satisfactory results in the current higher education theory and practice research.

This paper describes the running state and development level of higher education from the perspective of the synergy of scale effect and quality effect, which provides a new research method for higher education system.

\section{THEORETICAL CONSTRUCTION OF "QUANTITY-QUALITY- EFFECT" IN HIGHER EDUCATION}

\section{A. The basic concept of "quantity-quality-effect"}

As we know, the core of higher education research is how to solve the problem of education quality. This "quality" is not a single quality concept, but a systematic extension property. This is because the higher education system is a subsystem of the socio-economic system, in which there are three elements that can comprehensively reflect the state level of the higher education system, namely, the measurement of the quantity, quality and effect of higher education, referred to as the measurement of the "quantity-quality-effect " of higher education. The establishment of any educational model is confronted with the problem of establishing core values and theories. On the basis of comprehensive analysis of factors affecting the scale and quality of higher education, we try to explore solutions to problems, form a public security higher education mode that can meet the needs of the talent market and the development of students' individual potential, 
rationally guide the practical activities of higher education, and realize the overall improvement of higher education quality. In addition, under the trend of higher education reform and innovation, new ideas and methods and theoretical systems should be introduced to solve the problem of incompatibility between higher education research and practice.

The purpose of higher education research is to constantly improve the construction mode, operation mechanism and the relationship and function between higher education and social and economic development. Although the theoretical research on higher education has been quite mature, more research achievements have been made in the simple and universal research on the scale and quality of higher education. However, there is still a certain distance in the application of social practice, and there is a lack of research on the essential characteristics of higher education from the perspective of multiple synergies. Therefore, it presents the phenomenon of international higher education quality crisis.

The theoretical significance of credibility research lies in that it breaks away from the traditional educational research framework and establishes a new research methodology from the perspective of multi-disciplinary and mutual information based on mesoscopic scale. In fact, higher education research always focuses on the two themes of "scale effectiveness" (quantitative effectiveness) and "quality effectiveness" (qualitative effectiveness), and "quantitative effectiveness" and "quality effectiveness" are interdependent and influence each other in the operation of the education system. Therefore, the comprehensive criterion of "quantitative efficiency" and "qualitative efficiency" can fully reflect the operation state of the higher education system, and we call this evaluation criterion "quantity-quality-effect". The research shows that the core of the research on "quantity-quality-effect" of higher education is to seek a system mechanism of credible cooperation.

\section{B. Measurement of "quantitative effect" and "quality effect"}

Based on the characteristics of scale research of higher education in China, this paper establishes the concept of credible evaluation of higher education system from the perspective of the coordination and harmony of scale effectiveness (" quantity effectiveness") and quality effectiveness (" quality effectiveness "), and puts forward the theoretical research framework of "quantity-quality -effect " of higher education. Using the new self-organization theory of system non-optimal analysis, this paper analyzes and discusses the credible scale and quality of higher education system, and establishes the research method of the credible level of higher education system.

The research shows that the trusted scale of higher education system is characterized by the production attribute of school scale, the utility attribute of demand scale and the adaptive attribute of contradiction transformation. In addition, the general quality of higher education personnel training system is a quality of availability, the quality of specialized personnel training is a quality of performance, and the quality of specialized qualified personnel training is a quality of efficiency. The significance of credible quality lies in transforming the performance of education quality into the efficiency of education quality, so as to realize the multiobjective cooperative purpose of talent cultivation in higher education system.

We know that the "quantity effect" and "quality effect" of higher education represent the trusted scale and quality in the trusted education system. As the higher education system is a complex system, its complexity is mainly characterized by incomplete information reflecting the attributes of the education system. Therefore, the maximum value of scale credibility and quality credibility is 1 . If let $T(g)=$ scale credibility, $T(z)=$ quality credibility, then:

$$
\lim _{g \rightarrow x} T(g)=1, \lim _{z \rightarrow y} T(z)=1 .
$$

That is to say, the "quantity effect" and "quality effect" of higher education are sub-optimal, and the goal of higher education reform and innovation is to seek the maximum credible scale and maximum credible quality, that is, to obtain the maximum sub-optimal level of higher education system. The credible scale of higher education (" quantitative effect ") is particularly important to improve the comprehensive level of the higher education system. The scale of higher education cannot meet the needs of national economic or social development, or exceeds the carrying capacity of the higher education system itself, which will lead to the decline of higher education level. It can be said that without sufficient scale as the basis, quality improvement is out of the question, but if the scale is expanded to a certain extent, without good coordination and control, quality will be affected. Therefore, there is a strong interdependence and complementarity between the characteristics of quantity and quality in higher education, which determines the existence and evolution of the comprehensive characteristics of quantity and quality.

\section{CREDIBLE COOPERATIVE MECHANISM OF HIGHER EDUCATION}

\section{A. Concordance of higher education system operation}

According to the nature, attribute and purpose of higher education, and in combination with the characteristics of contemporary social and economic development, the core problem of higher education research is how to meet the needs of social and economic development, that is to say, to play a synergistic role of higher education in social and economic operation and development. In fact, it can be fully explained from the research on higher education and social and economic development that it is this "synergy" that determines the nature, attribute and development goal of higher education. Theory of higher education system in recent years studies have shown that the higher education system is a non-equilibrium self-organization system, the education system of the characters of open, collaborative, blurred characteristics has become a hot spot of research, research approach has been out of the traditional mode of education, education research is more focused on higher education system output the reasonable evaluation of social contribution. From the 
perspective of system science, the basic characteristics of higher education system are determined by the inevitability of education dynamics and the randomness of education statistics.

In fact, it is entirely possible to evaluate the running state of the higher education system from the aspects of "quantity effect" and "quality effect" of higher education, that is to say, use the characteristic space of "quantity-quality-effect" to evaluate the collaborative characteristics of the higher education system. In order to study the rationalization, we will scale system, quality system as the subsystem of the higher education evaluation system, then, for the higher education system, in which case when the system control parameters ("quantity effect ", "quality effect ") to demand point during the development of social economy, higher education system will appear what kind of evolution characteristics, called the research problem of higher education synergetic (in order to adapt to the back of the study, here only put forward the basic idea, the complete research will be introduced in other research literature). From the perspective of the higher education evaluation system composed of two subsystems (relatively speaking), the operation of each subsystem is bound to be related to each other, which may involve constraints on each other or push each other interdependent. The degree of harmony between the two subsystems can be divided into three levels, namely, coordination, collaboration and harmony. From the perspective of system science and system engineering, the synergy of higher education system can be evaluated by different states of eigenvalues of , and can be quantitatively expressed by "Concorde", as shown in table 1:

TABLE I CONCORDANCE OF HIGHER EDUCATION SYSTEM OPERATION

\begin{tabular}{|l|c|l|l|l|l|}
\hline \multirow{2}{*}{ Cooperation level } & \multicolumn{2}{|c|}{ Non-optimum } & \multicolumn{2}{c|}{ Optimum } \\
\cline { 2 - 6 } & Against & Restriction & Coordinate & Synergy & Harmonious \\
\hline Criterion & $T(g)+T(z) \ll 1$ & $T(g)+T(z)=1$ & $T(g)+T(z) \rightarrow 2$ & $T(g)+T(z)=2$ & $T(g)+T(z)>2$ \\
\hline Concordance degree & $<-1$ & {$[-1,0)$} & {$[0,1)$} & 1 & $>1$ \\
\hline
\end{tabular}

The concord development of higher education is the operational goal of higher education system. The new concept of concord development proposed by us has the following meanings: first, concord development refers to the reasonable and reasonable development of the internal and external elements of the education system in order to make the "quantity, quality and efficiency" meet the social needs. To correctly understand the connotation of harmonious development, we must first understand the concept of "concord" and "development". As mentioned above, Concorde has three system features. The first is the coordination feature, which means to solve conflicts or conflicts through some means or measures. This system feature exists as a process, so it is called process feature. The second is the collaborative feature, whose significance lies in the integration relationship between internal and external elements of the education system through the coordination process. At this time, it indicates a credible state of the development of the education system, so it can be called the status feature. The third is the harmonious characteristic, which is the target characteristic under the credible behavior of the education system. In addition, according to An Unabridged, Comprehensive Dictionary, development refers to "the change process of things from small to large, from simple to complex, from low to high, and from old quality to new quality". It can be seen that: first of all, the main body of "concordant development" of the education system has diversity. Secondly, "concord development" emphasizes a dynamic nature, in which there is both quantitative and qualitative change effect. Third, the results of "concordant development" are uncertain and complex, so the research on it belongs to the higher education synergies, which is also the future work of the researchers of this project.

\section{B. Research method based on meso-scale}

At present, researches on higher education based on scale and quality are mostly reflected in the macroscopic scale, mainly describing the running state and development trend of national or regional higher education. Because the complexity of higher education is manifested in the micro scale, the information is missing if the research mode is adopted, and the theoretical and practical analysis results of higher education are greatly deviated and difficult to be applied in practice. Therefore, it is necessary to adopt a scientific research scale that conforms to the complexity characteristics of higher education, namely the meso-scale research method.

Multi-scale phenomenon is a universal phenomenon in nature and human society. In the fields of physical science, life science, ecology and environment science and social science, multi-scale phenomenon can be found everywhere, which is closely related to complexity science. In the process of complex system analysis and evaluation, the boundary division of research field will affect the choice of measurement scale, and there is a correlation between different scales. In other words, how do you get properties on the small scale from properties on the large scale? What are the details of its process? This directly affects people's understanding of the development and evolution of complex systems, which is the problem of meso-scale. It is a common challenge for scientists and researchers to understand mesoscale processes and establish the interrelation between large and small scales. In fact, all problems can be considered as discrete (individual) on small scale, and show continuous average (population distribution) characteristics on large scale. Mapping from small scale to large scale, we will find completely different phenomena and rules. Moreover, how the properties of these individuals affect the average properties of large scale under relevant conditions, and what are the details of its process, which directly affects people's in-depth understanding of the development and evolution rules of 
complex systems. In addition, the research perspective of the traditional higher education system is based on the overall demonstration and analysis in the macro environment. Therefore, the research results lose a lot of information, which makes the analysis conclusion of many educational characteristics in the blind space. In other words, the data related to the operation of the education system becomes a kind of "blind number". This project adopted the basic method of "meso-scale" to reduce the influence of "blind number" in education statistics.

The theory of fitness landscape is a theory developed in the 1930 s, which is based on the biological view that species evolve continuously for survival, and evolution can be regarded as a journey on a three-dimensional landscape with peaks and valleys. Each site in the landscape represents a possible genetic combination, with a high degree of fitness for survival. There are many peaks and valleys in the landscape, different heights are related, slightly different combinations are close to each other and have similar fitness. In this way, the peaks are adjacent to each other and the valleys are adjacent to each other, giving the landscape a landscape of alternating mountains and valleys, which is called the fitness landscape. Later, the theory of fitness landscape was used to study physical problems, and gradually extended to other complex systems. Since the 1990s, the theory of fitness landscape has been applied in the fields of management and economics, and has been recognized by organizations and management experts. Since the beginning of this century, it has become a key concept to understand the structure and interaction of complex systems, and has played an important role in social science research of sociology and pedagogy. Higher education fitness landscape theory to study the main content of the fitness function is effective to establish education system, for example, whether it's scale and the design and evaluation of education quality education, to some extent, corresponds to the fitness function, can in the study on the characteristics of the public security institutions of higher education, establish the concept of scale and credible quality, in fact is a kind of fitness function, also is a kind of higher education built fitness landscape research methods.

The fitness landscape of higher education system proposed in this study completely describes the extension attribute of "quantity, quality and efficiency" of higher education from the perspective of system. Its purpose is to analysis the credibility of higher education in the social and economic development, in the interpretation of the sustainable development in society, higher education should play an important role, the key lies in the higher education system must be based on social extension properties of self-organization system, and measure "quality effect" is the effective criterion of self-organization system. Because of different social fields of higher education system has its own education and social attribute, at the same time, this study discusses the higher education from the perspective of higher education theory of "quality effect", for the higher education reform and development plan to provide certain theoretical basis, at the same time, also provide certain reference for other higher education research. The adaptation landscape of higher education based on "quantity-qualityeffect" is reflected in the relationship between colleges and universities, the educated and the social needs. Adaptability of higher education is composed of "quantity and effect" and "quality and effect" of higher education. The quality of higher education refers to the sum of the process and results of higher education to meet the obvious or implicit needs of individuals, groups and society. The quality of higher education is the life of higher education, and the ultimate expression of the use of educational resources by the educational population. The scale of higher education is the inevitable result of the main body of higher education. The level of higher education is the practical effect produced by the practice of higher education. It clearly reflects the needs and interests of the subject and deeply reflects the value relationship between higher education activities and people themselves. As shown in Fig. 1:

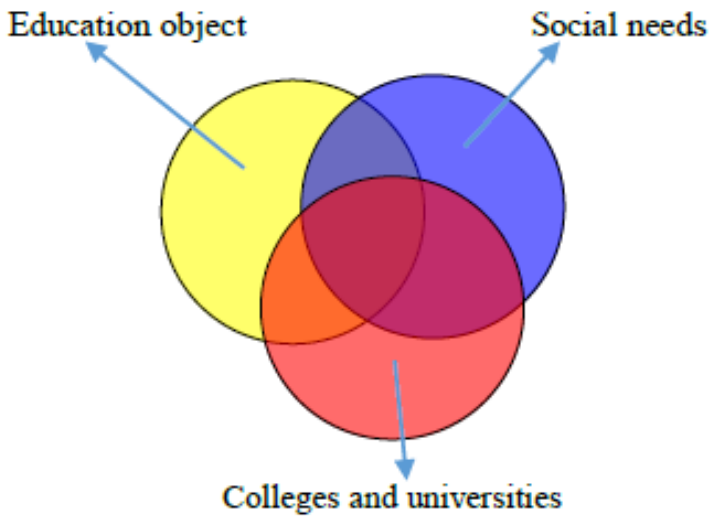

Fig.1. Relationship between subject, object and society in higher education

\section{CONCLUSIONS}

In this study, the higher education system is regarded as a trusted collaborative system with self-organization characteristics, and the adaptability landscape of higher education can be judged according to the concordancy of higher education in terms of "quantity, quality and efficiency". The results show that the adaptability landscape of higher education reflects the distribution characteristics of the development level of higher education and its contribution to society.

\section{REFERENCES}

[1] Yang Huan. A Study on the Coordination Theories and Methods for High Educational Sustainable Development System and Their Applications. Tianjin University, 2005. (In Chinese)

[2] Trow, Martin. "Reflections on the Transition from Elite to Mass to Universal Access: Forms and Phases of Higher Education in Modern Societies since WW II" in James J.F. Forest and Philip Altbach (eds.) International Handbook of Higher Education, Netherlands: Springer. 2006, pp243-280

[3] Zeng Jian, Zhang yifang. Social synergetics. Science Press, 2000. (In Chinese)

[4] He Ping. Extension Character and Cooperative Development Model of Police Higher Education in China. JOURNAL OF LIAONING POLICE ACADEMY. 2011, pp.1-6. (In Chinese)

[5] He Ping, Research on Trusted scale theory and practice of public security higher education. JOURNAL OF LIAONING POLICE ACADEMY, 2012, no.1, pp.1-7. (In Chinese)

[6] Li JInqi, He Ping, Credible quality theory and quality evaluation of public security higher education. JOURNAL OF LIAONING POLICE ACADEMY, 2012, no.5, pp.1-7. (In Chinese) 
[7] Miao Hong, Li Quansheng, Wu Jianw ei1. An Analysis and Forecast of the Size of Higher Education of China. Journal of China University of Geosciences (Social Sciences Edit ion). 2004, Vol.4, pp.75-77. (In Chinese)

[8] Luo Shengquan, Zhao Zheng. Use and Significance of Synergetics in Education Science Study. Journal of Mianyang Normal University. 2004, vol.23, pp.44-47. (In Chinese) 